\title{
In-Vitro Antioxidant Activity, Acute Oral Toxicity Studies and Preliminary Phytochemical Characterization of the Bark Extract of Terminalia arjuna (L.)
}

\author{
Samadhan G. Patil ${ }^{1}$, Bhushan S Bhadane ${ }^{1}$, Mohini P. Patil ${ }^{1}$, Sateesh Belemkar ${ }^{2}$ and \\ Ravindra H. Patil ${ }^{1, *}$ \\ ${ }^{1}$ Department of Microbiology and Biotechnology, R. C. Patel Arts, Commerce and Science College, Shirpur \\ 425405 (MS), India \\ ${ }^{2}$ Department of Pharmacology, SVKM NMIMS, School of Pharmacy and Technology Management, Shirpur \\ 425405 (MS), India
}

\begin{abstract}
The free radicals and the reactive oxygen species (ROS) are known to induce oxidative stress and it has been implicated in the pathology of cardiovascular diseases, inflammatory conditions, cancer and ageing. The activities associated with ROS can be delayed, prevented or removed by antioxidant compounds (natural or synthetic). The use of synthetic antioxidants restricted because of their known side effects such as liver damage and carcinogenesis. The aim of this study to evaluate in vitro antioxidant and acute oral toxicity of Terminalia arjuna extracts. The present finding reveals that the purified fraction at $100 \mu \mathrm{g} / \mathrm{ml}$, showed maximum $(91.32 \pm 0.10 \%$,) DPPH radical scavenging effect in comparison with standard ascorbic acid $(79.46 \pm 0.10 \%)$ at the $10 \mu \mathrm{g} / \mathrm{ml}$ concentration. The reducing power of the purified extract was found to be dose dependent. Food and water intake of the animals in test and control groups was found normal during the 14 day acute oral toxicity studies and no apparent changes were observed in the internal organs of both, the test and control groups, after gross necropsy. The preliminary phytochemical screening of the crude acetone extract revealed dominant presence steroids, terpenoids, polyphenols, alkaloids and tannins. TLC profile of the purified fraction revealed a single band of $R_{f} 0.38$, a characteristic feature of triterpenoids. The UV absorption maximum of the purified fraction was recorded at 194nm. The FT-IR spectrum indicated presence of aromatic rings $3421(\mathrm{COOH}), 2957$ (alkanes, $\mathrm{CH}_{2}$ and $\mathrm{CH}_{3}$ ), 1726 (carbonyl), 1599 (carboxylic acid), and region between 1000-1300 stretching of C-O, ester and ether carboxylic group. Thus, the isolated bioactive phytoconstituents form the bark extract of Terminalia arjuna could be used as natural anti-oxidants.
\end{abstract}

Keywords: Antioxidant activity, Acute oral toxicity, T. arjuna, TLC, Triterpenoids.

\section{INTRODUCTION}

Free radicals and reactive oxygen species (ROS) generated in the biological system are the major cause of the degenerative conditions such as aging, cancer, inflammation and atherosclerosis [1]. Hydroxyl radical $\left(\mathrm{OH}^{-}\right)$, superoxide anion $\left(\mathrm{O}_{2}^{-}\right)$and nitric oxide $\left(\mathrm{NO}^{-}\right)$are known to cause membrane damage, protein denaturation and lipid peroxidation. Natural antioxidant enzymes present in the body as well as dietary intake of antioxidants circumvents free radicals and reactive oxygen species generated in the body. Antioxidants also have the ability to prevent the oxidative stress generated by the reactive oxygen species and consequently the free radical mediated oxidative damage in the cell [2]. Sufficient amounts of exogenous antioxidants are required to reduce the damage caused by free radicals and reactive oxygen species. Therefore, there is great need to search for safe, less

*Address correspondence to this author at the Department of Microbiology and Biotechnology, R. C. Patel Arts, Commerce and Science College, Shirpur 425405 (MS), India; Tel: +91 2563 257328; Fax: +91 2563 257329; E-mail: ravi_nmu@yahoo.co.in cytotoxic and chemo-preventive natural antioxidants. Antioxidant potential of medicinal plants attributed by the bio-active compounds present in it. Medicinal plants are rich source of diverse secondary metabolites having potential to reduce reactive oxygen species and thus widely recognized for their pharmaceutical and medicinal importance. A number of clinical studies have reported that antioxidants of plant origin such as polyphenols, flavonoids, terpenoids, tannins etc. are reported to reduce the oxidative stress in the biological system [3].

Indian traditional system of medicine has identified a large numbers of plants for their antioxidative potential and human diseases. Medicinal plants such as Celatrus peniculatus, Carrisa carrandus, Achyrahes aspera, Cassia auriculata, Coccinia indica, Mentha spicata, Hygrophilla auriculata, Datura stramonium, Delonix regia, Coriandrum sativum and Pterospermum acerifolium are known to have antioxidant activities [4]. Terminalia arjuna (Combretaceae), commonly known as Arjun tree, is a large deciduous tree known for its cardioprotective role. The bark of $T$. arjuna has been recommended and used as a cardiac tonic and bark 
powder/decoction is used to treat heart diseases, bone fractures, skin diseases, poly-urea, white discharge, giddiness, fever and worms.

The phytochemistry of $T$. arjuna is well understood. The bark powder is known to contain tannins, triterpenoids (arjunic acid and arjungenin) and terpenoids glycosides like arjunnetin and arjunglucoside [5]. Several experimental and clinical studies have been undertaken and proved the beneficial effect of the T.arjuna bark in ischemic heart disease and cardiovascular complications [6]. However, safety and the mechanism of action of arjuna phytochemicals is uncertain. The present study demonstrates the in vitro antioxidant activity as well as evaluates the safety and toxicity by studying the acute oral toxicity of various extracts of T.arjuna. The study also aims at isolation and partial characterization of the bioactive fraction using chromatographic and spectroscopic techniques.

\section{MATERIALS AND METHODS}

\section{Plant Material and Preparation of Extract}

The bark of plant $T$. arjuna was collected from Toranmal Hill Station (MS, India). Plant material was identified and authenticated by the expert taxonomist and the voucher specimen (RCP-09/2014) was deposited in Department of Botany R. C. Patel Arts, Science College Shirpur. The bark collected from healthy plant was cut to obtain thin slices and subjected for shade drying at room temperature. The shade dried bark ground in a mechanical grinder to obtain a fine powder. The bark powder was further subjected to successive extraction in the Soxhlet extractor using solvents with increasing polarity. The organic phase of the extract was collected using separating funnel to obtain crude extract. The extract from separating funnel was filtered using Whatman filter paper No. 1 and subjected to dryness in rotary vacuums evaporator (Equitron, India) at high pressure and stored in refrigerator until used.

\section{Antioxidant Activity Using DPPH Radical Scavenging Assay}

The antioxidant activity was evaluated by means of measuring the 1,1diphenyl 2-picrylhydrazyl (DPPH) radical scavenging activity of the test extracts [8]. The method is based on reduction of DPPH in the presence of hydrogen-donating antioxidant due to the formation of the non- radical form of DPPH-H. This conversion indicated by color change from purple to yellow, it was measured spectrophotometrically at $517 \mathrm{~nm}$ [10]. Briefly, freshly prepared $1 \mathrm{ml}$ of methanolic DPPH (2 $\mathrm{mM}$ ) was mixed with different concentration of extract and ascorbic acid standard (Vitamin C), The mixture was shaken vigorously and incubate for $30 \mathrm{~min}$ in the dark, and the absorbance was measured at $517 \mathrm{~nm}$ against a blank. The percent DPPH radical scavenging ability was calculated using the following formula.

DPPH radical scavenging ability $(\%)=\left(A_{0}-A_{1}\right) / A_{0} \times$ 100

Where, $A_{0}$ is the absorbance of control, $A_{1}$ is the absorbance of sample. All samples were analyzed in triplicate.

\section{Reducing Power Assay}

The test extracts with different concentrations were mixed with $0.2 \mathrm{M}, 2.5 \mathrm{ml}$ phosphate buffer $(\mathrm{pH} \mathrm{6.6)}$ and $1 \%, 2.5 \mathrm{ml}$ potassium ferricyanide. The mixture was incubated for $20 \mathrm{~min}$ at $50^{\circ} \mathrm{C}$ and cooled, and then $10 \%, 2.5 \mathrm{ml}$ tricarboxylic acid was added and the mixture was centrifuged for $10 \mathrm{~min}$ at $600 \mathrm{~g}$. The upper layer of the solution $(2.5 \mathrm{ml})$ was mixed with $2.5 \mathrm{ml}$ of distilled water and $0.1 \%, 0.5 \mathrm{ml}$ ferric chloride $\left(\mathrm{FeCl}_{3}\right)$. The absorbance was measured at $700 \mathrm{~nm}$. [8].Ascorbic acid and phosphate buffer were used as standard and blank, respectively. All samples were analyzed in triplicate.

\section{Acute Oral Toxicity}

The purpose of acute oral toxicity testing of the plant and their preparations influence their safety to human, particularly for a pharmaceutical preparation. Acute oral toxicity testing was performed according to the OECD guideline no 423 as per the CPCSEA approval (SPTM-IAEC/Dec 14/7/18). Young, healthy 78 week Albino mice were employed for the testing and the animals were fasted for $3-4 \mathrm{~h}$ before dose. A single dose of crude extract and purified fraction of $T$ arjuna at 300,1000 and $2000 \mathrm{mg} / \mathrm{kg}$ body weight was given orally. After administration of dose, the food was withheld for 1-2h. For feeding, conventional animal diet was used and animals were kept in the separate polypropylene cages. Animals were observed for clinical signs of toxicity at $0-0.5,0.5-1,1-2,2-4,24-48 \mathrm{~h}$ post dosing with special attention during first $4 \mathrm{~h}$. If no mortality was observed for $48 \mathrm{~h}$, other mice were administered with dose of $1000 \mathrm{mg} / \mathrm{kg}$ body weight and observed for same symptoms. In the absence of toxicity, mice were administered with dose $2000 \mathrm{mg} / \mathrm{kg}$ body weight and checked for mortality or toxicity. Cage 


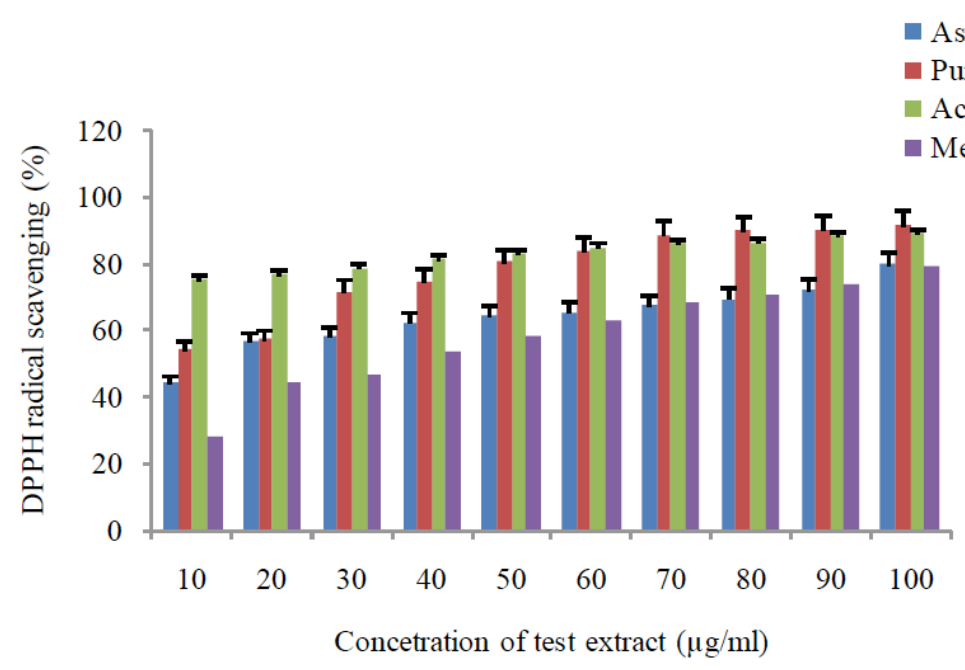

Figure 1: \% DPPH radical scavenging effect of acetone, crude extract, and purified fraction and standard (ascorbic acid). Data are shown as mean $\pm S D$ of values obtained in three separate experiments.

side observation includes changes in skin fur, eyes and mucous membrane (nasal), respiratory rate, heart rate and blood pressure, salivation, lacrimation, perspiration and CNS changes.

\section{Isolation and Characterization of Bioactive Metabolites}

The crude acetone extract after drying in vacuum afforded a brown solid which re-dissolved in methanol. The preliminary phytochemical investigation of the test extracts was carried out according to previously reported methods [9]. The resulting extract was subjected for Thin-Layer Chromatography (TLC). Five microliters of sample was applied using Spraylin-V applicator (Aetron, India) on aluminum TLC plate coated with Silica gel $\mathrm{G}_{\mathrm{F} 254}$ (Merck, India) and developed in ascending mode using different mobile phases. For separation of phytoconstituents, the extract was dissolved in acetone and adsorbed on silica gel of 60-120 mesh size for column chromatography. The air dried slurry was applied onto the top of column which was preconditioned with hexane. The column was eluted with hexane (100\%), hexane: ethyl acetate $(80: 20,60: 40$ and $50: 50 \mathrm{v} / \mathrm{v})$; chloroform: methanol (70:30, 50:50, and 20:80 v/v) and finally with $100 \%$ methanol. The fractions were collected separately and matched by TLC fingerprinting for tentative identification and homogeneity.

\section{UV and FT-IR Spectral Analysis}

The purified fraction obtained from silica gel column was subjected for UV and FT-IR spectroscopic characterization. The UV spectrum of purified fraction eluted from the column was recorded between 200-700 $\mathrm{nm}$ by UV-Visible spectrophotometer (Shimadzu 1750, Japan). FT-IR spectrum of the dried powder was recorded by FT-IR spectrophotometer (Shimadzu 8202 $\mathrm{PC}$, Japan $4000-400 \mathrm{~cm}^{-1}$ ) using $\mathrm{KBr}$ as a matrix.

\section{RESULTS}

\section{Antioxidant Activity by DPPH Radical Scavenging Assay}

The DPPH radical scavenging activity of the acetone crude extract, purified fraction and ascorbic acid was found to be dose dependent and the results are shown in Figure 1. The crude acetone extracts of $T$. arjuna showed $88.80 \pm 0.10 \%$ scavenging effect. The results showed that, the purified fraction at 100 $\mu \mathrm{g} / \mathrm{ml}$, showed maximum (91.32 $\pm 0.10 \%$ ) scavenging effect in comparison with standard ascorbic acid (79.46 $\pm 0.10 \%)$. The $\mathrm{IC}_{50}(\mu \mathrm{g} / \mathrm{ml})$ of the crude acetone extract and purified fraction of $T$. arjuna was found to be $140 \pm$ 11.21 and $45.95 \pm 5.74$, respectively (Table 1 ).

Table 1: IC $_{50}$ of DPPH Radical Scavenging Activity

\begin{tabular}{|c|c|}
\hline Activity & IC $_{50}(\boldsymbol{\mu g} / \mathbf{m l})$ \\
\hline \hline Purified fraction & $45.95 \pm 5.74$ \\
\hline Acetone crude extract & $140 \pm 11.21$ \\
\hline Methanol crude extract & $79.37 \pm 8.55$ \\
\hline Ascorbic acid & $6.9 \pm 0.4$ \\
\hline
\end{tabular}

\section{Reducing Power Assay}

The results of reducing power of the acetone and methanol crude extracts, purified fraction and ascorbic 


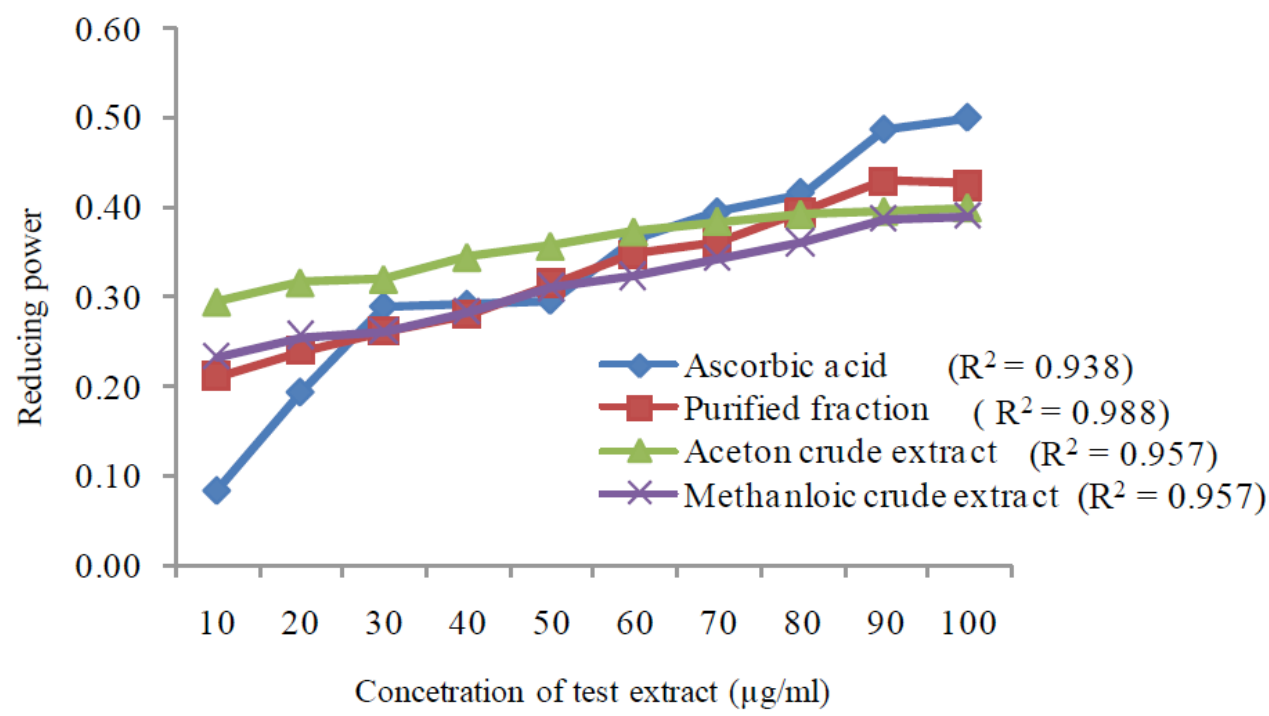

Figure 2: Reducing power of acetone crude, methanol crude, purified fraction and ascorbic acid data shown as mean \pm SD.

acid standard are shown in Figure 2. Reducing power of the purified fraction was found to be dose dependent $\left(R^{2}=0.988\right)$. The results indicate that reducing potential of the purified fraction is significantly higher than that of acetone; methanol crude extract and is comparable with the ascorbic acid standard. The results clearly demonstrate the admirable reducing power potential of purified fraction. The bioactive constituents present in the extracts may have potential donating activities which lead to their strong reducing potential.

\section{Acute Oral Toxicity}

The results of acute oral toxicity studies are summarized in Tables 2 and $\mathbf{3}$. The food and water intake of the animals in test and control groups was found normal (Table 3). During the 14 day oral acute toxicity testing, no mortality, no sign of toxicity and negative symptoms was observed in any animals treated with purified fraction of the T.arjuna. There is no statistically significant difference $(P<0.05)$ was observed in water and food intake between vehicle control and test group (Table 3 ). The changes in body weight at 300,1000 , and $2000 \mathrm{mg} / \mathrm{kg}$ body weight of T.arjuna purified fraction was not significant (Figure 3 ). The results of acute treatment effect of the $T$. arjuna purified fraction on organ weight in mice are shown in Table 2. No apparent changes were observed in the organs of both, the test and control groups, after gross necropsy.

\section{Chromatographic and Spectroscopic Characteriza- tion of the Active Principle}

The preliminary phytochemical screening of the crude acetone extract revealed dominant presence of phytoconstituents such as steroids and terpenoids, polyphenols, alkaloids and tannins. The crude extract of $T$. arjuna was subjected for column chromatography using optimized solvent system (non-polar to polar mobile phases) to elute out bioactive components. The column eluted with chloroform: ethyl acetate (1:1) and a total of 100 fractions (20 ml each) were collected. The fraction number thirty to sixty affording semisolid colorless compound does not showed significant

Table 2: Acute Treatment Effect of T.arjuna Purified Fraction on Organ Weight

\begin{tabular}{|c|c|c|}
\hline \multirow{2}{*}{ Organ } & \multicolumn{2}{|c|}{ Weight in grams } \\
\cline { 2 - 3 } & Control & T. arjuna purified fraction \\
\hline \hline Lungs & $1.89 \pm 0.11$ & $1.84 \pm 0.20$ \\
\hline Liver & $1.42 \pm 0.25$ & $1.29 \pm 0.26$ \\
\hline Spleen & $0.19 \pm 0.28$ & $0.15 \pm 0.24$ \\
\hline Heart & $0.14 \pm 0.15$ & $0.17 \pm 0.10$ \\
\hline Kidney & $0.43 \pm 0.26$ & $0.41 \pm 0.35$ \\
\hline
\end{tabular}

Data expressed mean $\pm S D$. 
Table 3: Food and Water Intake During Acute Toxicity Study with Purified Fraction

\begin{tabular}{|c|c|c|}
\hline Parameters & Control & T. arjuna purified fraction \\
\hline \hline Water (mL/day) & $6.98 \pm 0.57$ & $7.15 \pm 0.48^{\mathrm{a}}$ \\
\hline Food (g/day) & $2.91 \pm 0.35$ & $3.25 \pm 0.27^{\mathrm{a}}$ \\
\hline
\end{tabular}

Data are expressed as mean $\pm S D$. $P<0.05$, are considered for statistically significant between groups.

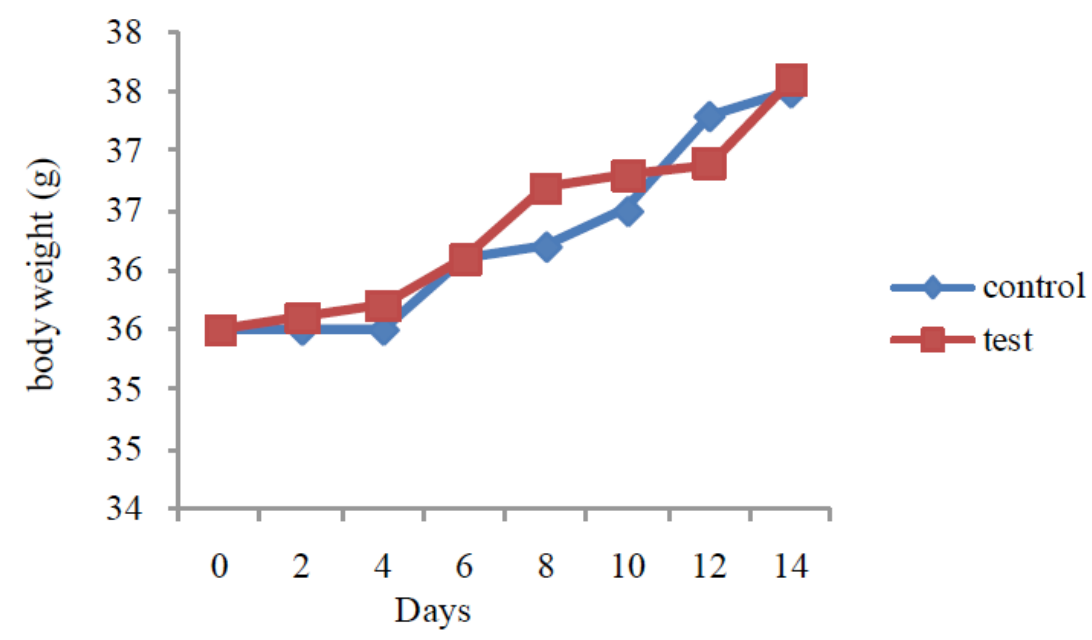

Figure 3: Changes in the body weight after administration $T$. arjuna purified fraction.
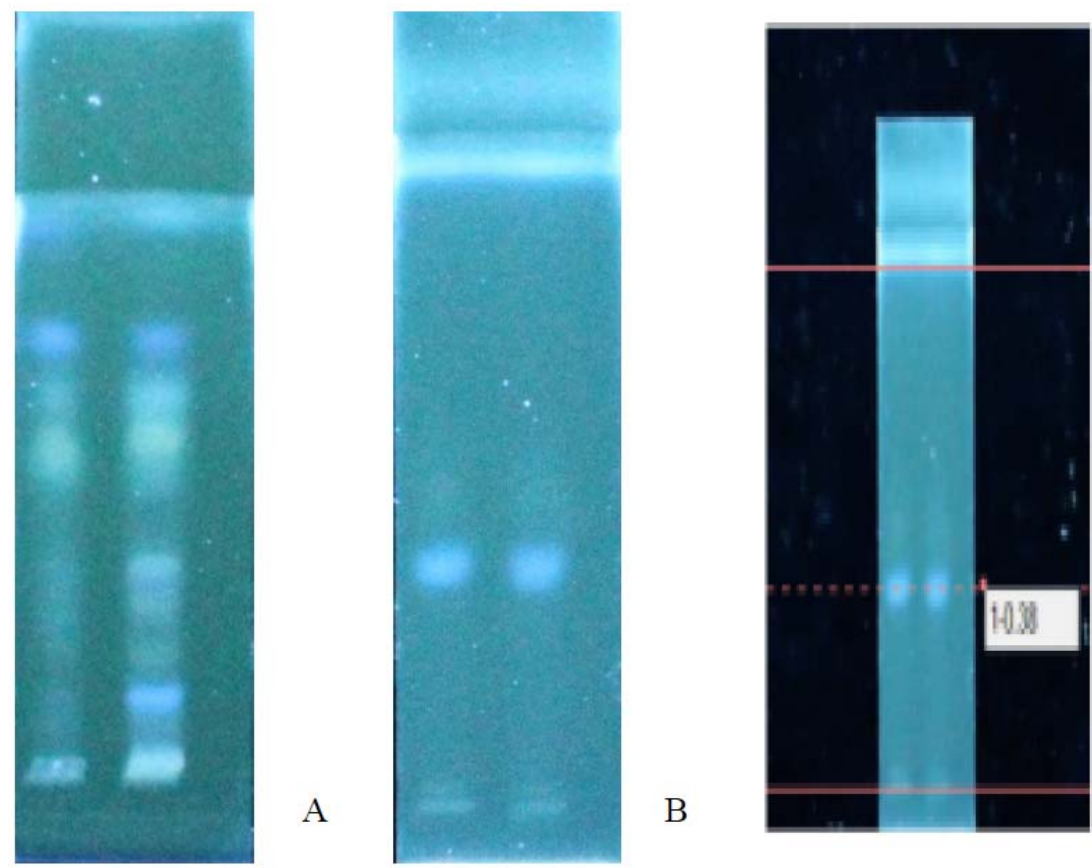

C

Figure 4: TLC profile of T.arjuna (A) Crude extract (B) Purified fraction, $\mathrm{R}_{\mathrm{f}}$ of purified fraction (C).

bioactivity (data not shown) and hence discarded. Fraction number sixty five to hundred afforded a colorless solid showing single band of $R_{f} 0.38$ (Figure 4C) in different mobile phases showed excellent bioactivity hence subjected to further characterization using spectroscopic and chromatographic techniques.
Among the various mobile phases tried for TLC separation, better separation was obtained with chloroform: ethyl acetate $(1: 1 \mathrm{v} / \mathrm{v})$ when $5 \%$ sulfuric acid was used as spraying reagent (Figure 4A). The TLC separated band of purified fraction showed fluorescence at longer wavelength (Figure 4B) which is 
A

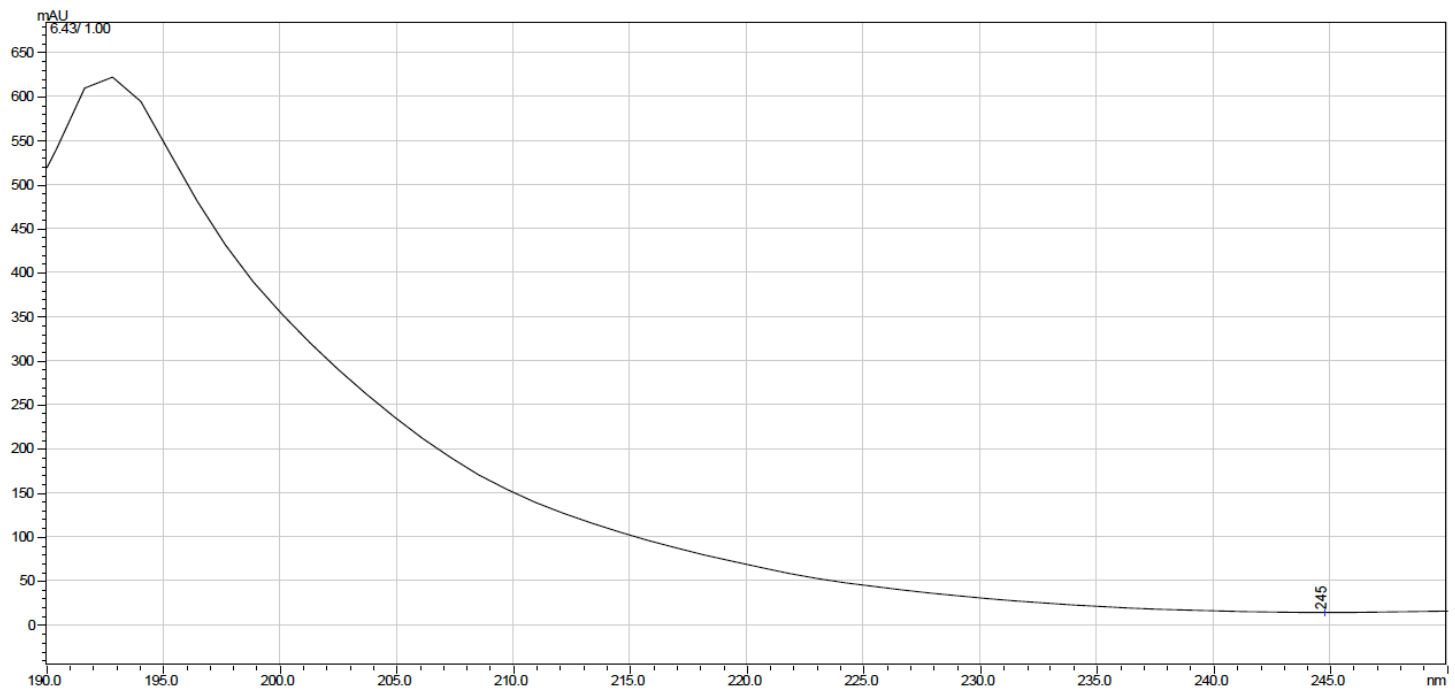

B

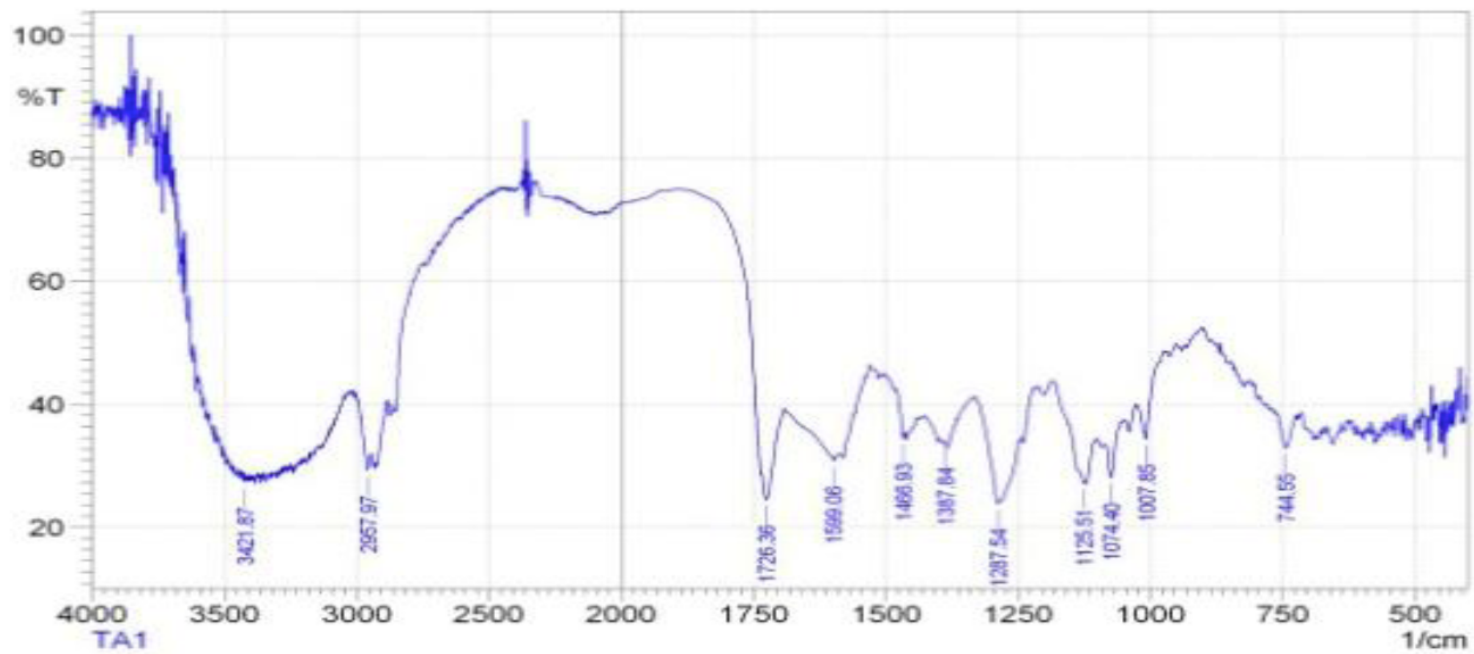

Figure 5: UV (A) and FT-IR (B) spectrum of the purified fraction of T. arjuna.

the characteristic feature of triterpenoids. UV spectrum of purified fraction of $R_{f} 0.38$ showed maximum absorption at $194 \mathrm{~nm}$ (Figure 5A). The FT-IR spectrum (Figure 5B) showed presence of aromatic rings. A stretching at $3421(\mathrm{COOH}), 2957$ (alkanes, $\mathrm{CH} 2$ and $\mathrm{CH}_{3}$ ), 1726 (carbonyl), 1599 (carboxylic acid), and region between 1000-1300 (C-O, ester and ether carboxylic group) was observed. On the basis of TLC characteristics, UV and FT-IR spectrum, the purified fraction may contain terpenoids as a major constituent.

\section{DISCUSSION}

Natural products from plant sources have various protective and therapeutic effects and they are essential for preventing various diseases [11]. Many Indian medicinal plants such as Borago officinalis, Capparis sicula subsp. Sicula, Malva sylvestris, Mentha aquatic, and Raphanus raphanistrum have been explored as natural source of strong antioxidants [12]. Our study demonstrated the strong antioxidant potential and acute oral toxicity of the bark extract of $T$. arjuna. Different physiological and pathological conditions generate large number of free radicals reactive oxygen species and reactive nitrogen species. Free radicals adversely affect lipids, proteins, and DNA and trigger a number of human diseases including aging and atherosclerosis. Thus a balanced intake of dietary antioxidants circumvents free radicals mediated damage. The antioxidant metabolites from $T$. arjuna in our studies are effective yet safe and thus can be effectively used as to prevent oxidative stress mediated cellular damages. Moreover, the $\mathrm{IC}_{50}$ value of purified fraction $\left(\mathrm{IC}_{50}=45.95 \pm 5.74 \mu \mathrm{g} / \mathrm{ml}\right)$ was found less than previous reports $[12,13]$. 
Natural antioxidants have ability to prevent the formation of ROS by donating electron and thus they prevent the oxidative DNA damage and lipid peroxidation (Rice-Evans et al. 1997). Thousands of medicinal plants available that contain phytochemicals with an excellent antioxidant potential. Plant secondary metabolites like phenolics and flavonoids with antioxidant activity are widely studied. These compounds play an important role to adsorb and neutralize free radicals, and quench singlet and triplet oxygen.

However, relatively few reports are exists on terpenoids. Moreover, lack of knowledge on safety and toxicity of the plant metabolites is the major constrain for their use in humans. The purified fraction in our studies showed no sign of toxicity and its maximum tolerated dose is more than $2000 \mathrm{mg} / \mathrm{kg}$ body weight. However further repeated acute toxicity study is required for the validation of purified fraction as safer, nontoxic antioxidant. Also, in-vivo clinical trials are required for the proof of its strong antioxidant activity. It can be concluded that, the purified fraction of $T$. arjuna has excellent free radical scavenging activity and reducing power and it was found better than standard, ascorbic acid. The bioactive principle of the purified fraction of $T$. arjuna could be terpenoids which are safe and could be used as natural source of antioxidants for providing alternative remedy for the management of oxidative stress.

\section{ACKNOWLEDGEMENT}

This research was supported by the University Grant Commission (UGC File No. 42-456/2013 SR) New Delhi-India.

\section{COMPETING INTEREST}

Authors declare that there are no conflicts of interest.

\section{REFERENCES}

[1] Yang D, Wang Q, Jiang J, Ying T. Antioxidant activities of various extracts of lotus (Nelumbo nuficera Gaertn) rhizome. Asian Pac J Clin Nutr 2007; 16: 158-163.
[2] De Brum TF, Zadra M, Piana M, Boligon AF, Rohlich J, Freitas D, Stefanello S, Forbrig A, Belke BN, Silva LR, Machado M, Da Rocha J, Soares F, Athayde M. HPLC analysis of phenolics compounds and antioxidant capacity of leaves of Vitex megapotamica (Sprengel) moldenke. Molecules 2013; 18: 8342-8357.

https://dx.doi.org/10.3390/molecules 18078342

[3] Ali G. Flavonoids and phenolic acids: role and biochemical activity in plants and human. J Med Plants Res 2011; 5: 6697-6703.

https://dx.doi.org/10.5897/JMPR11.1404

[4] Kirtikar KR, Basu BD. Indian Medicinal Plants Lalit Mohan Basu, Allahabad 1918 pp. 75-80.

[5] Dwivedi S. Terminalia arjuna Wight \& Arn- A useful; drug for cardiovascular disorders. J Ethanopharma 2007; 114: 114129.

https://dx.doi.org/10.1016/j.jep.2007.08.003

[6] Rane MM, Mengi SA. Comparative effect of oral administration and topical application of alcoholic extract of Terminalia arjuna bark on incision and excision wounds in rats. Fitoterapia 2003; 74: 553-558.

https://dx.doi.org/10.1016/S0367-326X(03)00118-7

[7] Mary NK, Babu BH Padikkala J. Antiatherogenic effect of Caps HT2, an herbal Ayurvedic medicine formulation. Phytomed 2003; 10: 474-482.

\section{https://dx.doi.org/10.1078/094471103322331412}

[8] Isabel F, Paula B, Miguel V, Lillian B. Free-radica scavenging capacity and reducing power of wild edible mushrooms from north east Portugal: Individual cap and stipe activity. Food Chem 2007; 100: 1511-1516. https://dx.doi.org/10.1016/j.foodchem.2005.11.043

[9] Olayinka A, Anthony I. Preliminary phytochemical screening and In vitro antioxidant activities of the aqueous extract of Helichrysum longifolium DC. BMC Complemen Alter Med 2010; 10: 21.

https://dx.doi.org/10.1186/1472-6882-10-21

[10] Oktay M, Gulcin I, Kufrevioglu I. Determination of in vitro antioxidant activity of fennel (Foeniculum vulgare) seed extracts. LWT - Food Sci Tech 2003; 36: 263-271. https://dx.doi.org/10.1016/S0023-6438(02)00226-8

[11] Mishra SS. Patel KK, Raghuvanshi N, Pathak A, Panda PP, Kundlik G, Patro N. Screening of ten Indian medicinal plant extracts for antioxidant activity. Annals Bio Res 2011; 2: 162170.

[12] Filomena C, Silvio S, Mariangela M, Federica M, Giancarlo A.S, Dimitar U, Aurelia T, Francesco M, Roberto DL. In vivo anti-inflammatory and in vitro antioxidant activities of Mediterranean dietary plants. J Ethnopharmacol 2008; 116: 144-151. http://dx.doi.org/10.1016/j.jep.2007.11.015

[13] Bushra S, Faroog A, Roman P. Antioxidant activity of phenolic components present in barks of Azadirachta indica, Terminalia arjuna, Acacia nilotica, and Eugenia jambolana Lam. Trees. Food Chem 2007; 104: 1106-1114. http://dx.doi.org/10.1016/j.foodchem.2007.01.019 